\title{
Efficacy and Safety of Sansoninto in Insomnia with Psychiatric Disorder: An Open-Label Study
}

Tsuyoshi Miyaoka*, Kiminori Kawano, Motohide Furuya, Rei Wake, Sadayuki Hashioka, KristianLiaury, Erlyn Limoa, Keiko Tsuchie, Michiyo Fukushima, Tomoko Araki and Jun Horiguchi

Department of Psychiatry, Shimane University School of Medicine, Izumo, Japan

*Corresponding author: Tsuyoshi Miyaoka, Department of Psychiatry, Shimane University School of Medicine, 89-1 Enyacho, Izumo 693-8501, Japan, Tel: 853-20-2262; Fax: 853-20-2260; E-mail: miyanyan@med.shimane-u.ac.jp

Rec date: Aug 01, 2014; Acc date: Dec 30, 2014; Pub date: Jan 01, 2015

Copyright: @ 2015 Miyaoka T, et al. This is an open-access article distributed under the terms of the Creative Commons Attribution License, which permits unrestricted use, distribution, and reproduction in any medium, provided the original author and source are credited.

\section{Abstract}

Background: Prior research confirms that insomnia is highly prevalent in patients with psychiatric disorders. Benzodiazepine hypnotics, causing serious disadvantages, have been widely used in psychiatry for a long time. Sansoninto (SNT), Japanese herbal medicine, is used for patients with weakness and fatigue, annoyance, insomnia, amnesia, and neurotic symptoms.

Objective: The efficacy and safety of SNT was examined in adult psychiatric disorder patients with insomnia symptoms.

Methods: Eighty-one adults with sleep disturbance meeting DSM-IV-TR diagnostic criteria for psychiatric disorders (schizophrenia: 17; monopolar depression: 20; bipolar depression: 10; adjustment disorder: 12; anxiety disorder: 5; others: 17) were treated openly for four weeks with SNT (2.5-7.5 g) at bedtime. Patients maintained sleep throughout the study. Efficacy was analyzed using a repeated measures methodology. The primary outcome was the Pittsburgh Sleep Quality Index (PSQI). The secondary outcomes were the Insomnia Severity Index (ISI), Athens Insomnia Scale (AIS), Clinical Global Impression-Improvement (CGI-I), and change of dosage of benzodiazepine hypnotics (diazepam equivalent).

Results: After 4 weeks of SNT therapy, significant symptom reduction was observed on all parameters (PSQI: $10.22 \pm 3.23$ vs. $3.11 \pm 3.52$; ISI: $20.63 \pm 4.86$ vs. $3.38 \pm 5.10$; AIS: $17.41 \pm 4.69$ vs. $2.85 \pm 4.23$; dosage of benzodiazepine hypnotics [diazepam equivalent, mg]: $10.5 \pm 4.71$ vs. $2.98 \pm 3.37$ ). No withdrawal involved treatmentrelated adverse events.

Conclusion: Data from this 4-week open-label study suggests SNT was an effective and generally well tolerated treatment for insomnia symptoms in this sample of adult patients with psychiatric disorders.

Trial Registration: controlled-trials.com Identifier: UMIN000014156.

Keywords: Psychiatric disorder; Sunsoninto (SNT); Insomnia; Japanese Herbal Medicine

\section{Introduction}

Sleep disturbances are common in individuals with psychiatric disorders [1]. For example, sleep problems are present in approximately $80 \%$ of patients with a major depressive disorder (MDD) [2], in $30 \%$ to $80 \%$ of patients with schizophrenia [3], and in at least $55 \%$ of individuals with active substance abuse [4]. Although these sleep disturbances often are secondary to the psychiatric illness, recent observations strongly suggest that sleep disorders should be actively treated parallel to psychiatric disorders. The few studies regarding this matter indicate that separate treatment of comorbid sleep disturbances exerts positive effects on the course of the psychiatric disorder and may prevent relapse [5]. Sleep disturbance is not only one of the most common symptoms of psychiatric disorders, but also one of the major determinants relating to quality of life (QOL) for patients with psychiatric disorders [6-8].
Benzodiazepines quickly gained acceptance as the preferred treatment for insomnia after appearing on the pharmaceutical market in 1960. Despite clinical guidelines recommending their use [9], there are still claims that the benefits of this older generation of hypnotics are outweighed by serious disadvantages including daytime sedation, dependence, rebound insomnia, slurred speech, staggering gait, poor judgment, and slow uncertain reflexes. Not surprisingly, many patients with sleep difficulty have turned to Japanese herbal medicine (kampo medicine) to manage their sleep problems and improve their QOL.

Kampo medicines use natural substances that generally have low toxicity and few side effects, treats disease as unique to the individual, and balance homeostasis and increasing immunity to diseases. Among traditional prescriptions used to treat sleep disorders, sansoninto (SNT; suazaoretang), a well-known traditional sedative, is used for patients with weakness and fatigue, annoyance, insomnia, amnesia, and neurotic symptoms [10]. In the classical literature, SNT is said to 'nourish the blood' and calm the nerves to eventually bring on a tranquilizing sensation and reduce the effect of sleep disturbance. SNT is a combination of five medicinal herbs: Jujube seed (Zizyphi Semen), 
Citation: Miyaoka T, Kawano K, Furuya M, Wake R, Hashioka S, et al (2015) Efficacy and Safety of Sansoninto in Insomnia with Psychiatric

Page 2 of 6

Poria sclerotium (Poria), Cnidium rhizome (Cnidii Rhizoma), Anemarrhena rhizome (Anemarrhenae Rhizoma) and Glycyrrhiza (Glycyrrhizae Radix) [11] (Table 1).

In experimental studies, SNT showed the hypnotic effect on sleep enhancement and its underlying mechanisms may be mediated through the activation of serotonergic system in additional to the activation of gamma-amino butyric acid A (GABAA) receptors in rat $[12,13]$. At the global metabolomics levels, SNT can increase sleep activity and exhibit binding affinity for serotonaergic receptors in a model of insomnia drosophilia [14]. Jujube seed, as the principal drug in the SNT, has exhibited the central nervous system tranqualizing effect. Spinosin has been proposed as the active component in Jujube seed [15]. In addition, the hypnotic effect of Jujube seed may be mediated by the anticholinergic and antihistamine action of betulic acid, an active compound of Jujube seed $[16,17]$.

SNT is also available in the United States, but as an over-thecounter dietary supplement. Despite its popularity, no scientific evidence to back up claims of efficacy or safety in clinical use of SNT. Pharmaceutical companies are generally reluctant to conduct any randomized controlled trial for SNT, which is already on the market and approved by regulatory bodies. However, it is unethical not to conduct clinical trials to outline the safety profile of SNT; clinical trials will also provide basic knowledge to reveal possible mechanisms of
SNT's effectiveness. An observation period of four weeks can start to expose any unknown side effects and may verify an ancient tradition that SNT is effective in improving sleep quality, sleep latency, sleep disturbance, sleep efficiency, and daytime function in patients with psychiatric disorders.

The objective of this four-week open study in a naturalistic setting was to evaluate the efficacy and safety of SNT in patients with psychiatric disorders.

\section{Method}

\section{Herbal formula}

The SNT formula used in this study is an herbal extract powder produced according to the good manufacturing procedures (GMP) of the certified company Tsumura Pharmaceutical Co., Ltd. (Japan), for the purpose of meeting international market standards of quality and uniformity. Apart from providing SNT, Tsumura Pharmaceutical was not involved in any other sponsorship, study design, or monitoring of the participants. In Japan, SNT has been approved by the Ministry of Health, Labour and Welfare, and is covered by the National Health Insurance plan. The compounds were classified on the basis of the constituent herbs of SNT on a chromatogram (Table 1).

\begin{tabular}{|l|l|}
\hline Constituent of SNT & Compounds \\
\hline JP Juijube seed (Zizyphi Semen) & Sanjoinine A \\
\hline JP Poria sclerotium (Poria) & Dehydropachymic acid \\
\hline JP Conidium rhizome (Chidii Rhizoma) & Geissoschizine methyl ether, hirsuterine, hirsutine \\
\hline JP Anemarrhena rhizome (Anemarrhenae Rhizom) & Anemarsaponin Alll, Anemarsaponin C \\
\hline JP Glycyrrhiza (Glycyrrhizae Radix) & $\begin{array}{l}\text { Formononetin, formononetin-7-O-glucoside } \\
\text { Liquiritigenin, liquiritin, liquiritin apioside, glycyrrhizin, glycyroside, isoliquiritin apioside, } \\
\text { isoliquiritin, isoliquiritigenin, glycylcoumarin }\end{array}$ \\
\hline
\end{tabular}

Table 1: Classification of compounds identified in the three-dimensional chromatogram.

\section{Preparation of herbs}

All herbal components of SNT were prepared in large computercontrolled boiler which collected volatile oils and herbal broth, pumped into a vacuum drying condenser, and sprayed onto a base material. These granulated compounds were then vacuum-dried at low temperatures before being siphoned into a separate sterile-room, where they were packaged, labelled, and sealed. Granules were packed in aluminium foil packages and are administered orally at a dose of 2.5-7.5 g once per day. For quality assurance of the active ingredients in SNT, HPLC (high performance liquid chromatography) fingerprinting was employed to identify substances in the final product (Figure 1). No animal products, endangered species, or restricted herbal ingredients were used in this study. Each batch of SNT was also tested for E. coli, Salmonella (bacteria count), and heavy metals.
Figure 1

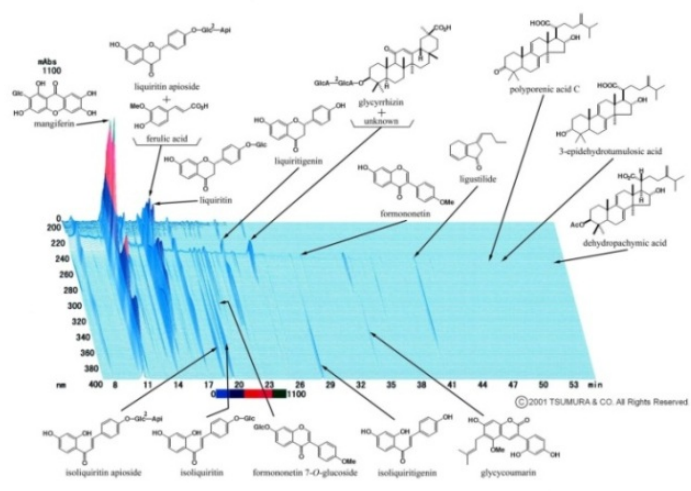

Figure 1: High performance liquid chromatography. 


\section{Recruitment of subjects}

Patients of the Department of Psychiatry of the Shimane University Hospital were enrolled in the study. The study obtained approval from the Shimane University Hospital Institutional Review Board (No. 1067). Participants were recruited between April 2011 and April 2012. All participants understood the nature of the trial and willingly signed the informed consent form.

\section{Eligibility criteria}

The subjects enrolled in the study were patients with psychiatric disorders meeting DSM-IV-TR criteria [18], ranging from 27-80 years of age, with a Pittsburgh sleep quality index (PSQI) of greater than six. These patients spent 45 minutes or more falling asleep, slept less than six hours, and had struggled with this issue continuously for at least one month.

\section{Ineligibility criteria}

Patients with psychiatric disorders were ineligible to participate in the study if (1) they had had a major incident within three months of the study, (2) were working the nightshift, (3) lacked time or had timing conflicts, (4) had confounding disease such as obstructive sleep apnea-hypopnea, restless legs syndrome, cardiac arrthythmia, acute myocardial infarction, high blood pressure, diabetes, or cancer, (5) were currently taking pharmaceuticals that affect melatonin, acetylcholine, adenosine or prostaglandins, (6) were unable to read and fill out the forms for the study, or (7) had any evidence of renal or liver dysfunction as defined by a level of at least 1.5 times of the upper reference limit (serum creatinine: $1.3 \mathrm{mg} / \mathrm{dL}$, blood urea nitrogen: 22 $\mathrm{mg} / \mathrm{dL}$, serum aspartate-aminotransferase (AST): $25 \mathrm{IU}$, alanineaminotransferase (ALT): 29 IU/L).

\section{Study design and procedure}

Psychiatrists, physicians, and nurses were briefed on important points prior to the start of the study and attended a training session to ensure their duties were conducted in accordance with good clinical practice guidelines. Participants underwent an initial screening phase of two visits. On the first visit, they were interviewed to obtain information about their medical history and were later subjected to a clinical examination. The second visit determined the baseline data on symptoms and included a basic health assessment that consisted of complete blood counts and biochemical function tests. These tests were performed to ensure the eligibility of each patients and to identify respondents with potential poor compliance. At the end of the second visit, each eligible patient was provided with sufficient SNT for a treatment dose of 2.5-7.5 g once daily (at bed-time) to take until the next visit. No other herbs were allowed during the study period.

Follow-up visits were scheduled for the first and fourth weeks of the study. Physical examinations, basic health assessments, and biochemical function tests for each patient were carried out the end of the study. At the end of the trial, the participants received a further physical examination, including blood tests. Patients were contacted by telephone one to two days prior to each visit to encourage their continued participation. Throughout the study, leftover packages were counted to monitor each patient's compliance. The occurrence of adverse effects (AEs) was recorded on every visit.

\section{Efficacy}

The primary outcome parameter evaluated prior to and following the four week intervention was the PSQI. The PSQI is a multidimensional, self-administered, health status measure that probes clinically important, patient-relevant symptoms in the areas of sleep quality and quantity. The PSQI comprises a 19-item questionnaire with seven sub-scales (subjective sleep quality, sleep latency, sleep duration, habitual sleep disturbances, sleep efficiency, use of sleep medication, and daytime dysfunction) [18]. Each subscale is rated 0-3 with the higher scores reflecting more severe sleep complaints. The sum of the scores indicates the patient's overall sleep experience. The lower the overall score, the better the person sleeps. The tool possesses adequate internal reliability, validity, and consistency for clinical and community samples of psychiatric patients [19], However, the questions are heavily based on memory over the past month. Therefore, the study included four secondary outcome parameters: the Insomnia Severity Index (ISI) [20], Athens Insomnia Scale (AIS) [15], Clinical Global Impression-Improvement (CGI-I) [21], and dosage of benzodiazepine hypnotics (diazepam equivalent) [22].

\section{Safety assessment}

To set up an active safety surveillance system, we first surveyed the traditional medicine practitioners currently using SNT in the treatment of sleep difficulty to ascertain any AEs these practitioners had noticed. They reported that such medication occasionally resulted in gastrointestinal discomfort such as acid regulation, heartburn, and abdominal discomfort when SNT was administered orally at a dose of 2.5-7 g, once per day. We included these on our list for surveillance. We also included the AEs most frequently reported by the National Reporting System: abdominal fullness, diarrhoea, vomiting, nausea, urticaria, itching, purpura, jaundice, skin vesicles (or local reddish swelling), edema, hypotension, bradycardia, dyspnoea, fever, muscle cramps, and sleepiness on our surveillance list. Other possible AEs were enquired about as the 21 st item. All subjects were required to take objective laboratory tests to detect any abnormal liver, blood, or kidney findings at the baseline and after four weeks of SNT therapy.

\section{Statistical analysis}

Our analysis focused mainly on changes in the seven-domain constructs of the PSQI assessment. The study described the treatment effects as changes in mean differences among the participants between the baseline scores and those measured at visits on weeks one and four. Separate linear regression models were constructed for the response variables of differentiated scores on subjective sleep quality, sleep latency, sleep duration, habitual sleep disturbances, use of sleep medication, and daytime dysfunction subscales. The explanatory variables in the final regression model were age, marital status, educational level, and baseline severity of psychiatric symptoms. To examine improvement across the month, two indicator variables for visits at weeks one and four were also included in the regression model. The coefficients of the two indicator variables represent the differences between the average values of measured scores for the visits at weeks one and four versus those at baseline. We assumed correlated error terms among the repeated measurements for each participant. Finally, each single PSQI score item was also examined separately to identify any PSQI items sensitive to treatment by SNT. All of the above analyses were performed using the SAS (9.2 edition) software package. On the basis of data from previous reports, we calculated the required sample size to be 75 [5]. 


\section{Results}

\section{Study population}

Of 99 sample patients, 18 were deemed to be ineligible. The principal reasons for ineligibility were a disinterest in participation $(\mathrm{n}=12)$, medical conditions and medications $(\mathrm{n}=3)$, failure to show up for further study $(\mathrm{n}=2)$, and lack of evidence of severe sleep difficulty $(n=1)$. In all, 81 of the initial participants intending to treat (ITT) completed the 4 -week study without any major protocol violation. For safety analysis, full analysis set testing was performed.

Table 2 summarizes the baseline demographics and clinical characteristics of the study. The patients in the study had a mean age of $44.5 \pm 8.4$. All subjects were diagnosed with insomnia associated with psychiatric disorder according to International Classification of Sleep Disorders Second Edition (ICSD-2) [23], and classified as: schizophrenia, 17; monopolar depression, 20; bipolar type I, 4; bipolar type II, 6; adjustment disorder, 12; anxiety disorder, 5; and others, 17. At the start of SNT administration, all subjects were receiving BZD hypnotics (one kind of drug, 44; two or more kind of drugs, 37).

\begin{tabular}{|c|c|}
\hline \multicolumn{2}{|l|}{ Clinical Characteristics } \\
\hline Sex, n (male/female) & $35 / 46$ \\
\hline Age, mean $\pm S D$, year & $44.5 \pm 8.4$ \\
\hline \multicolumn{2}{|l|}{ Diagnosis } \\
\hline Schizophrenia & 17 \\
\hline Monopolar depression & 20 \\
\hline \multicolumn{2}{|l|}{ Bipolar affective disorder } \\
\hline Type I & 4 \\
\hline Type II & 6 \\
\hline Adjustment disorder & 12 \\
\hline Anxiety disorder & 5 \\
\hline Others & 17 \\
\hline \multicolumn{2}{|l|}{ Hypnotics } \\
\hline Diazepam equivalent (mg) & $10.5 \pm 4.7$ \\
\hline One drug & 44 \\
\hline Two or more drugs & 37 \\
\hline
\end{tabular}

Table 2: Demographic and clinical characteristics of 81 patients completing a 4-week trial of SNT.

\section{Efficacy}

After four weeks of treatment, there were significant improvements in each of the PSQI subscales. The magnitudes of improvement in the PSQI subscales were 30\% for subjective sleep quality, $22 \%$ for sleep latency, $27 \%$ for sleep duration, $9.3 \%$ for habitual sleep disturbances, and $27 \%$ for daytime dysfunction (Table 3 ). The mean amount of time to fall asleep per day was reduced from $60.6 \pm 46.0$ minutes at the baseline to $31.0 \pm 20.0$ minutes at the end of the study. The mean sleep duration per day was prolonged from $4.7 \pm 1.0$ hours at the baseline to $6.5 \pm 1.1$ hours after four weeks treatment. In other secondary outcomes, ISI, AIS, and CGI, significant improvement of symptoms was observed. The mean dosage of benzodiazepine hypnotics was reduced significantly after 4 -week treatment by SNT (Table 3 ).

\begin{tabular}{|c|c|c|c|}
\hline & Baseline & $\begin{array}{l}\text { After } 4 \text { weeks of } \\
\text { TJ-103 therapy }\end{array}$ & P-value \\
\hline & $($ mean $\pm \mathrm{SD})$ & (mean $\pm \mathrm{SD})$ & \\
\hline $\begin{array}{l}\text { Pittsburg Sleep } \\
\text { Quality Index }\end{array}$ & $10.22 \pm 3.23$ & $3.11 \pm 3.52$ & $<0.0001$ \\
\hline $\begin{array}{l}\text { Subjective sleep } \\
\text { quality }\end{array}$ & $2.56 \pm 0.57$ & $0.78 \pm 0.85$ & $<0.0001$ \\
\hline Sleep latency & $2.64 \pm 0.58$ & $0.58 \pm 0.74$ & $<0.0001$ \\
\hline Sleep duration & $2.33 \pm 0.92$ & $0.64 \pm 0.73$ & $<0.0001$ \\
\hline $\begin{array}{l}\text { Habitual sleep } \\
\text { efficacy }\end{array}$ & $2.14 \pm 1.12$ & $0.20 \pm 0.49$ & $<0.0001$ \\
\hline Sleep disturbance & $1.15 \pm 0.48$ & $0.33 \pm 0.50$ & $<0.0001$ \\
\hline Daytime dysfunction & $2.68 \pm 0.54$ & $0.58 \pm 0.89$ & $<0.0001$ \\
\hline $\begin{array}{l}\text { Insomnia Severity } \\
\text { Index }\end{array}$ & $20.63 \pm 4.86$ & $3.38 \pm 5.10$ & $<0.0001$ \\
\hline $\begin{array}{l}\text { Athens Insomnia } \\
\text { Scale }\end{array}$ & $17.41 \pm 4.69$ & $2.85 \pm 4.23$ & $<0.0001$ \\
\hline \multicolumn{4}{|l|}{ Hypnotics } \\
\hline $\begin{array}{l}\text { Diazepam } \\
\text { equivalent (mg) }\end{array}$ & $10.5 \pm 4.71$ & $2.98 \pm 3.37$ & $<0.0001$ \\
\hline Tablets & $1.46 \pm 0.95$ & $0.59 \pm 0.45$ & $<0.0001$ \\
\hline
\end{tabular}

Table 3: Major variables of baseline and week 4 measurements summarized as mean difference.

Among those ITT, psychiatric symptom scores of one of 81 patients had increased at the end of the study. Ten of these patients reported sleep quality at the same level as their baseline, one reported sleep quality worse than her baseline, and the remaining nine perceived better sleep quality by week four.

\section{Safety and adverse effects}

No tested biomarker demonstrated significant changes after the 4week SNT intervention. No participant withdrew from the study due to AEs. Intolerable side effects resulting in withdrawal included two events of stomach ache and nausea. We carried out periodic evaluation of any unexpected symptom during the treatment period and found the perception of gastrointestinal discomfort two days after SNT consumption, but it was dramatically relieved after SNT discontinuance. We were unable to rule out the possibility that ingesting SNT will cause the deterioration of pre-existing gastrointestinal symptoms.

\section{Discussion}

To the best of our knowledge, this study is the first to investigate the potential efficacy and safety of SNT in improving sleep quality in psychiatric disorders with sleep difficulty as measured by PSQI. Our results demonstrated a significant improvement in sleep quality of patients with psychiatric disorders after four weeks of SNT treatment. 
Although we used self-comparison to rule out potential confounding effects of BMI, smoking, exercise, and socioeconomic status, the possibility of placebo effect might still exist.

The present data showed that one week of SNT treatment had no effect, but there was significant improvement in sleep quality after a four-week treatment at a dose of 2.5-7.5 g once daily (Figure 2). From a physiologically based pharmacokinetic point of view, it takes more than one week to demonstrate the symptom-relief effect of the active ingredients in SNT, which appears to resemble a slow-acting drug, and it may not be appropriate to compare its action head-to-head with conventional medications of rapid action, such BZD hypnotics. The outcome of the evaluation study provides valuable evidence to avoid a direct comparison of the SNT formula with rapid action medications in future randomized controlled trials.

\section{Figure 2}

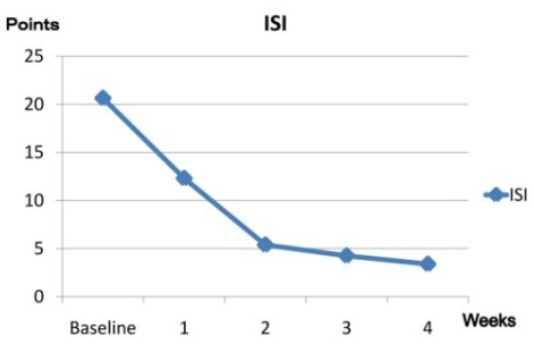

Figure 2: SNT treatment.

The dose of BZD hypnotics was reduced at almost the same time as improvement of the score, and the dose was further reduced with an improvement in the score. At week 4, the dose of BZD hypnotics had been reduced to about one third of that used at baseline. During the observation period, reducing the dose of BZD hypnotics did not cause any adverse events that may pose serious problems in medical practice, such as inter dose rebound insomnia.

Although an immediate effect of SNT cannot be expected due to its characteristics, the effect was observed after four weeks in the 34 subjects who switched to exclusive-use, and especially at week 2, 31 of the 34 subjects had been able to switch to SNT alone. This result suggests that continuous administration at least for one month would be ideal in order to reduce the dose of co-administered BZD hypnotics.

Generally, if the duration of administration of BZD hypnotics exceeds six months to one year, about $80 \%$ of patients experience withdrawal symptoms [24]. Therefore, as a method for gradual reduction of BZD hypnotics, reducing the daily dose about $25 \%$ every two to four weeks is recommended [25]. Most subjects in this study had taken BZD hypnotics for more than one year, and the speed of reduction was faster than recommended. However, because of the small number of subjects and the lack of evaluation of the effect of concomitant psychotropic drugs, it is difficult to assert that SNT has potential to prevent withdrawal symptoms.
With regard to the basic research examining the treatment of insomnia, current pharmacologic approaches have largely focused on the activity of $\gamma$-amino butyric acid A (GABAA), one of the inhibitory neurotransmitters in the central nervous system. In an experimental mouse model, it had been suggested that SNT has an activity different from that of synthetic hypnotics such as BZD. This is because SNT had no effect on pentobarbital (PB)-induced sleep in unstressed mice, and it reverses the stress-induced decrease and/or increase in PB sleep by improving stress-induced functional changes in the central nervous system rather than by acting like a synthetic hypnotic on GABAA receptors [26]. Yang et al. reported that SNT increases sleep activity and exhibits binding affinity for serotonin receptors. These results implicate the hypnotic effect of SNT, and its effects may be mediated through serotonergic activation. It provided strong evidence that the hypnotic effect of SNT occurred at the level of global metabolomics. By using a metabolomic approach, this study also demonstrates that metabolomics could provide a very promising way to elucidate the therapeutic mechanisms of Japanese herbal medicines [27].

Clinical trials have never explored the SNT dosage used in this study (2.5-7.5 g once daily). Without the present data, TM practitioners in Japan will continue to prescribe SNT to patients in their everyday practice based on documentation from a thousand years ago. The results of this study show (about IIb level) [15] that to participants had to withdraw from the study due to AEs, and that the two leading adverse drug reactions included stomach ache and nausea. The AEs discovered by this study, though not serious enough to prohibit the use of this formula clinically, show that TM practitioners must be alert to the possibility that prescribing SNT can cause severe adverse gastrointestinal effects. Further research to clarify the safety of SNT use is necessary [28].

The present study recruited psychiatric patients with poor sleep and used self-comparison to rule out the potential confounders of BMI, age exercise, and socioeconomic status. This type of study presents particular advantages. Limited subject numbers allow simple and comprehensive data analysis that can be used to identify possible trends, which a larger sample size could prove significant. Participants do not have to spend the extended amount of time necessary for a crossover design, an alternate method for dealing with a limited number of subjects. Additionally, this type of study avoids the ethical dilemma regarding giving a placebo to symptomatic participants who could be receiving valid treatment.

A major limitation of our study is its observational nature or lack of a randomized placebo group. Therefore, our claim of efficacy associated with SNT might not be as strict as that from a randomized clinical trial (RCT). No matter how common the use of SNT is in Japan, no RCT has been conducted yet. The evidence we obtained from the present study can provide important data on the safety profile of SNT and direct evaluation of its effectiveness, and meanwhile this study can serve as prior data for the design of RCTs. Another limitation of this study is that since participants were psychiatric patients with sleep problems in Japan, caution in generalizing these findings to patients in other nations is necessary.

\section{Limitation}

Given the design characteristics of this trial, the present findings should be taken cautiously. Study limitations include its open design and its small sample size. In an open-label study, both placebo effects 
and the effects of medication previously prescribed cannot be ruled out as explanations for the observed improvements.

\section{Conclusion}

Excluding psychiatric patients with a past history of stomach ache diarrhoea, and dizziness, the dosage form and prescription pattern of the SNT preparation appears to be well tolerated and valuable as a short-term alterative therapeutic option for improving daytime dysfunction in psychiatric patients with poor sleep quality during their psychiatric phase. A placebo effect, natural fluctuation, insufficient follow-up time, and/or other unobserved factors could confound the results of the current study. A prospective randomized, double-blind, controlled trial to further evaluate the efficacy of SNT on psychiatric patients with sleep difficulty is warranted in the future.

\section{Authors Contributions}

T. Miyaoka created the study design and coordination, patient recruitment, conceived the study, and helped draft the manuscript. M. Furuya conducted paper preparation and submission. R. Wake, S. Hashioka, K. Kawano, K. Liaury, E. Limoa, K. Tsuchie, M. Fukushima, and Jun Horiguchi were responsible for data acquisition and obtained the funding for the study. T. Araki and M. Fukushima performed all statistical analyses and interpretation of the data. All authors have read and approved the paper.

\section{Acknowledgments}

Part of this work was supported by Grant-in-Aid for Scientific Research on Priority Areas No. 13770544 and 50284047 from the Ministry of Education, Science, Sports and Culture and 012 from the Ministry of Health Labour Sciences Research Grant of Japan.

\section{References}

1. Abad VC, Guilleminault C (2005) Sleep and psychiatry. Dialogues Clin Neurosci 7: 291-303.

2. Tsuno N, Besset A, Ritchie K (2005) Sleep and depression. J Clin Psychiatry 66: 1254-1269.

3. Cohrs S (2008) Sleep disturbances in patients with schizophrenia : impact and effect of antipsychotics. CNS Drugs 22: 939-962.

4. Mahfoud Y, Talih F, Streem D, Budur K (2009) Sleep disorders in substance abusers: how common are they? Psychiatry (Edgmont) 6: $38-42$.

5. Smith MT, Huang MI, Manber R (2005) Cognitive behavior therapy for chronic insomnia occurring within the context of medical and psychiatric disorders. Clin Psychol Rev 25: 559-592.

6. Zammit GK, Weiner J, Damato N, Sillup GP, McMillan CA (1999) Quality of life in people with insomnia. Sleep 22 Suppl 2: S379-385.

7. Bélanger L, Morin CM, Langlois F, Ladouceur R (2004) Insomnia and generalized anxiety disorder: effects of cognitive behavior therapy for gad on insomnia symptoms. J Anxiety Disord 18: 561-571.

8. Riedel BW, Lichstein KL (2000) Insomnia and daytime functioning. Sleep Med Rev 4: 277-298.

9. National Institutes of Health (2005) National Institutes of Health State of the Science Conference statement on Manifestations and Management of Chronic Insomnia in Adults, June 13-15, 2005. Sleep 28: 1049-1057.
10. www.consensus.nih.gov/2005/2005InsomniaSoS026html.htm

11. Wang YC (1983) Hand book of Commonly Used Chinese Herbal Prescriptions, Oriental Healing Arts Institute, Long Beach CA

12. Ministry of Health (2006) Labour and Welfare, "The Japanese Pharmacopeia, Fifteenth Edition (15th edn)

13. Yi PL, Tsai CH, Chen YC, Chang FC (2007) Gamma-aminobutyric acid (GABA) receptor mediates suanzaorentang, a traditional Chinese herb remedy, -induced sleep alteration. J Biomed Sci 14: 285-297.

14. Yi PL, Lin CP, Tsai CH, Lin JG, Chang FC (2007) The involvement of serotonin receptors in suanzaorentang-induced sleep alteration. J Biomed Sci 14: 829-840.

15. Yang B, Zhang A, Sun H, Dong W, Yan G, et al. (2012) Metabolomic study of insomnia and intervention effects of suanzaoren decoction using ultra-performance liquid-chromatography/electrospray-ionization synapt high-definition mass spectrometry. J Pharm Biomed Anal 58: 113-124.

16. Li Y, Liang X, Xiao H, Bi K (2003) Determination of spinosin in rat plasma by reversed-phase high-performance chromatography after oral administration of Suanzaoren decoction. J Chromatogr B Analyt Technol Biomed Life Sci 787: 421-425.

17. Chen CY, Chen YF, Tsai HY (2008) What is the effective component in suanzaoren decoction for curing insomnia? Discovery by virtual screening and molecular dynamic simulation. J Biomol Struct Dyn 26: 57-64.

18. American Psychiatric Association (2000) Diagnostic and Statistical Manual of Mental Disorders, revision (DSM-IV-TR) (4thedn), American Psychiatric Association, Washington DC

19. Buysse DJ, Reynolds CF 3rd, Monk TH, Berman SR, Kupfer DJ (1989) The Pittsburgh Sleep Quality Index: a new instrument for psychiatric practice and research. Psychiatry Res 28: 193-213.

20. Bastien CH, Vallières A, Morin CM (2001) Validation of the Insomnia Severity Index as an outcome measure for insomnia research. Sleep Med 2: 297-307.

21. Soldatos CR, Dikeos DG, Paparrigopoulos TJ (2000) Athens Insomnia Scale: validation of an instrument based on ICD-10 criteria. J Psychosom Res 48: 555-560.

22. Guy W (1976) National Institute of Mental Health. CGI, Clinical Global Impression In ECDEU Assessment Manual for Psychopharmacology. revised Rockville MD: 534-537.

23. Inagaki A, Inada T (2006) Dose equivalence of psychiatric drugs. Jpn J Clin Psychopharmacol 9: 1443-1447.

24. American Academy of Sleep Medicine (2005) International Classification of Sleep Disorders (2nd edn) Diagnostic and Coding Manual. American Academy of Sleep Medicine Westchester IL

25. Rickels K, Case WG, Schweizer EE, Swenson C, Fridman RB (1986) Lowdose dependence in chronic benzodiazepine users: a preliminary report on 119 patients. Psychopharmacol Bull 22: 407-415.

26. Catena-Dell'Osso M, Marazziti D, Rotella F, Bellantuono C (2012) Emerging targets for the pharmacological treatment of depression: focus on melatonergic system. Curr Med Chem 19: 428-437.

27. Saito K, Umeda S, Kawashima K, Kano Y (2000) Pharmacological properties of traditional medicines. XXVI. Effects of Sansohnin-to on pentobarbital sleep in stressed mice. Biol Pharm Bull 23: 76-79.

28. WHO (2000) General guidelines for methodologies on research and evaluation of traditional medicines. Document: WHO/EDM/TRM/ 2000.1 Geneva, Switzerland. 